\title{
The Indentation Rolling Resistance in Belt Conveyors: A Model for the Viscoelastic Friction
}

\author{
Nicola Menga ${ }^{1,2}$, Francesco Bottiglione ${ }^{1, *(D)}$ and Giuseppe Carbone ${ }^{1}$ (D) \\ 1 Department of Mechanics, Mathematics and Management, Politecnico of Bari, V.le Japigia, 182, \\ 70126 Bari, Italy \\ 2 Department of Mechanical Engineering, Imperial College London, South Kensington Campus, \\ Exhibition Road, London SW72AZ, UK \\ * Correspondence: francesco.bottiglione@poliba.it
}

Received: 26 May 2019; Accepted: 2 July 2019 ; Published: 5 July 2019

\begin{abstract}
In this paper, we study the steady-state rolling contact of a linear viscoelastic layer of finite thickness and a rigid indenter made of a periodic array of equally spaced rigid cylinders. The viscoelastic contact model is derived by means of Green's function approach, which allows solving the contact problem with the sliding velocity as a control parameter. The contact problem is solved by means of an accurate numerical procedure developed for general two-dimensional contact geometries. The effect of geometrical quantities (layer thickness, cylinders radii, and cylinders spacing), material properties (viscoelastic moduli, relaxation time) and operative conditions (load, velocity) are all investigated. Physical quantities typical of contact problems (contact areas, deformed profiles, etc.) are calculated and discussed. Special emphasis is dedicated to the viscoelastic friction force coefficient and to the energy dissipated per unit time. The discussion is focused on the role played by the deformation localized at the contact spots and the one in the bulk of the thin layer, due to layer bending. The model is proposed as an accurate solution for engineering applications such as belt conveyors, in which the energy dissipated on the rolling contact of idle rollers can, in some cases, be by far the most important contribution to their energy consumption.
\end{abstract}

Keywords: rolling friction; indentation rolling resistance; viscoelasticity; friction; belt conveyors

\section{Introduction}

Polymers and rubber-like materials have found increasing utilization in mechanical devices and machines, because of some of their very relevant physical properties. Tires, seals, conveyor belts, power transmission belts, and seismic isolation devices are only a few examples of how important they are in commonly used mechanical systems. Rubber-like materials are characterized by a relatively high compliance (with respect to the metal materials they are usually coupled with), a large static and dynamic friction coefficient (specifically in the case of polymeric materials [1]), vibration absorption [2,3], resistance to some aggressive chemicals and environments [4], and much more. Since they are very often employed in contact pairs, there is a huge interest in deep understanding the details of the contact behavior of this kind of materials in static and dynamic conditions.

Their valuable characteristics, however, sometimes leads to some competing properties, thus the system design is critical. In belt conveyors, for example, rubber materials are used to protect the core of the belt (usually made of synthetic or metal fibers to bear the tensile load), preventing damages coming from aggressive environments and mechanical interactions, such as the rolling contact with the load-carrying idle rollers, as well as the abrasion induced by the conveyed material. As a side effect, while the belt is running, the rubber part of the belt undergoes time dependent cyclic deformation, which in turn leads to energy dissipation. A viscoelastic friction arises, which is often known as indentation rolling resistance [5]. Such power loss can be responsible for up to $60 \%$ of the total energy 
consumption in long horizontal conveying systems [6], thus making it critical to develop both accurate experimental techniques for the characterization of the belt cover material properties [7] and numerical approaches for accurately estimating the power loss in relation with the geometrical and operative characteristics of the conveyors systems. Indeed, a combination of a deep knowledge of the employed rubber compounds and an accurate modeling of the contact behavior is the basis of a smart design of such devices.

As far as contact modeling is concerned, the topic has been faced in the literature by analytical approaches [8-15], numerical simulations [16-21] and experiments [22-26]. Most of the literature about analytical or semi-analytical approaches to the study of the contacts involving rubber-like materials relies on the assumption that the contact bodies can be well represented by semi-infinite solids [12,27-29]. This hypothesis holds true for a large class of contact mechanics problems. As a rule of thumb, it can be considered that contact quantities are correctly calculated by modeling the bodies as half-spaces when the size of the contact area is sufficiently small compared to the thickness of the bodies (as in the Hertzian assumption [30]). However, the correctness of such approach breaks when dealing with contacts involving thin layers of deformable materials, either in the case they are backed to a stiffer body on the opposite side of the contact $[14,31,32]$, or in the case they are unconstrained [33,34]. Under such circumstances, proper methodologies must be developed. Because of the relative complexity in deriving rigorous analytical results, researchers have proposed some oversimplified approaches based on the viscoelastic Winkler foundation approximation of the deformable body $[35,36]$. Similarly, a very sophisticated theoretical formulation exists able to deal with such a demanding problem $[37,38]$, however, due to the fundamental mean-field nature of such a theory, its applicability to studies tailored to engineering applications is weak. As an alternative, semi-analytical models have been carried out [39,40]. In particular, in [40], the authors studied the sliding contact of viscoelastic layers of finite thickness on rigid sinusoidal substrates within the framework of Green's functions approach. They considered two different boundary conditions of the viscoelastic layer: confined and free layer. In [41], the authors exploited the results of the confined viscoelastic layer achieved in [40] to investigate the viscoelastic contact between rigid, equally spaced cylinders and a thin layer of rubber material constrained to a rigid substrate. They have characterized the viscoelastic friction arising in a moving contact, with the purpose of determining the damping behavior of a roller bearing viscoelastic isolator for seismic applications. Further, the effectiveness of such a semi-analytical approach has also been proved in a fundamental investigation on the rough contact behavior of viscoelastic layers [42], showing significant agreement with one of the most advanced closed-form theories on the topic $[11,43]$.

Moving from the formalism presented in [40], in this work, we investigate the viscoelastic rolling contact between a rubber belt and a row of rigid cylinders, spaced by equal intervals of length $\lambda$. An external uniformly distributed pressure is assumed to act on the opposite side of the belt, forcing the contact with the cylinders. Specifically, the rubber belt is assimilated to a free layer of linear viscoelastic material. The study allows determining the tangential viscoelastic frictional force (indentation rolling resistance) arising at the contact interface, as well as the energy dissipation per unit time, as functions of the system geometry, the mechanical properties of the viscoelastic layer and the operative conditions such as the layer velocity and the remote applied pressure.

\section{Physical Model}

A schematic picture of the physical system under investigation is shown in Figure 1. A thin layer of viscoelastic material is pushed against a row of equally spaced rigid cylinders by a uniform pressure distribution. The layer is kept in motion at constant velocity $V$ and we assume no friction at the interface between the layer and the cylinders. The viscoelastic "friction" is thus only the resultant of the horizontal components of the infinitesimal forces given by the contact pressure distribution between the cylinders and the layer. Indeed, a neat horizontal force on the belt arises as a consequence of the asymmetrical contact areas and pressure distribution. Under the contact mechanics perspective, 
it is equivalent to consider idle rollers with absent bearing resistance, or resistance to rotation of any other kind. This model can be applied as it is only in the practical cases in which the tangential stress distribution is weak enough not to alter considerably the stress and strain of the material in comparison with the frictionless case. Such a physical configuration resembles the case of a horizontal belt conveyor for granular materials, in which the belt, running at velocity $V$, is pressed against idle rollers by an almost uniform pressure due to the load of the granular material. The schematic picture in Figure 1 shows the contact penetration $\Delta$, defined as the distance between the mean line of the deformed lower surface of the viscoelastic layer and the upper point of the cylinder cross-section, whereas $u(x)$ is the layer interfacial displacement field. In our paper, we deal with linear viscoelasticity, thus we neglect any large deformation effect, as well as material response nonlinearity. This assumption may be quite restrictive in particular conditions, e.g., when the penetration $\Delta$ takes values close to the layer thickness $h$; nonetheless, it should not affect the main physical scenario and conclusions.

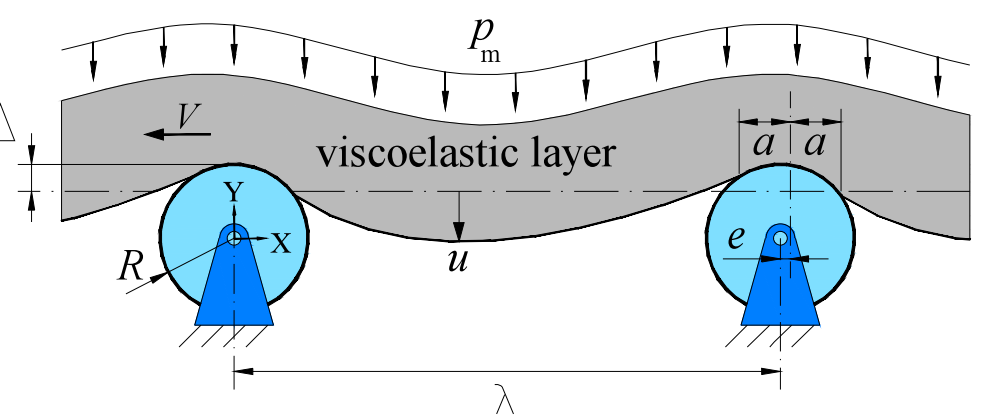

Figure 1. Schematic picture of the viscoelastic free layer pressed against the row of rigid cylinders. Annotations define the geometrical quantities relevant for formulation of the physical model.

The steady-state periodic viscoelastic contact problem depicted resembles the aperiodic rolling contact problem already treated semi-analytically by Hunter [5], and the periodic one studied by some of the authors of the present paper in [12] for the case of viscoelastic half-plane (i.e., $h \rightarrow \infty$ ). However, in the case of contacts with viscoelastic free layers of finite thickness, closed form solutions are not available, and a numerical procedure has to be adopted to treat the problem at hand. Here, we exploit the formalism firstly proposed in [20] and then extended to finite thickness free layers [40], which relies on a Green's functions approach. Therefore, under the assumption of steady moving at velocity $V$, the contact problem can be formalized in terms of an integral equation with a translational invariant kernel $\Theta_{V}(x-s)$, which parametrically depends on the velocity $V$

$$
u(x)=-\int_{\Omega} \Theta_{V}(x-s) p(s) d s
$$

where $\Omega=[-a, a]$ is the contact domain (see Figure 1). In Equation (1), the kernel $\Theta_{V}(x)$ represents the viscoelastic Green's function for steady moving contacts. Examples of $\Theta_{V}(x)$ can be found for single asperity contacts in [5], and periodic contact in [12]. In the case of periodic contacts with free layers of finite thickness, according to the authors of [40], it takes the form

$$
\Theta_{V}(x)=J\left(0^{+}\right) G(x)+\int_{0^{+}}^{+\infty} G(x+V t) \dot{J}(t) d t
$$

where $J(t)$ is the viscoelastic creep function, whose general form is 


$$
J(t)=\mathcal{H}(t)\left[\frac{1}{E_{0}}-\int_{0}^{+\infty} d \tau C(\tau) \exp (-t / \tau)\right]
$$

with $\mathcal{H}(t)$ the Heaviside step function, $E_{0}$ the zero-frequency elastic modulus of the viscoelastic material, and $C(\tau)$ the spectrum of elastic compliances. Further, the term $G(x)$ in Equation (2) is the so-called purely elastic Green's function. Such a shape function is strictly related to the specific geometry of the problem under investigation (see [44])

$$
\begin{aligned}
G(x)= & \frac{2\left(1-v^{2}\right)}{\pi} \log \left[2\left|\sin \left(\frac{k x}{2}\right)\right|\right] \\
& +\frac{2\left(1-v^{2}\right)}{\pi} \sum_{m=1}^{\infty} A_{m}(k h) \frac{\cos (m k x)}{m}
\end{aligned}
$$

where

$$
A_{m}(k h)=\frac{2 h k m-\sinh (2 h k m)}{1+2(h k m)^{2}+\cosh (2 h k m)}+1
$$

being $h$ the viscoelastic layer thickness, and $k=2 \pi / \lambda$. As a first example of application, the case of a linear viscoelastic material with a unique relaxation time $\tau$ is considered herein. In this case,

$$
J(t)=\mathcal{H}(t)\left[\frac{1}{E_{0}}-\frac{1}{E_{1}} \exp (-t / \tau)\right]
$$

with $1 / E_{1}=1 / E_{0}-1 / E_{\infty}$.

Substituting Equation (6) into Equation (2) and recalling that $J(+\infty)=1 / E_{0}$ and $J(0)=1 / E_{\infty}$, where $E_{\infty}$ is the high frequency elastic modulus, the long time response viscoelastic Green's function writes as

$$
\Theta_{V}(x)=\frac{1}{E_{\infty}} G(x)+\frac{1}{E_{1}} \int_{0^{+}}^{+\infty} G(x+V \tau z) \exp (-z) d z
$$

Moreover, it has been shown that the layer surface mean-plane displacement with respect to the underformed configuration is (see [40]):

$$
u_{\mathrm{m}}=\frac{1+v}{1-v} \frac{1-2 v}{E_{0}} p_{\mathrm{m}} h
$$

where $p_{\mathrm{m}}=\lambda^{-1} \int_{\Omega} p(x) d x$ is the mean contact pressure.

In Figure 1, it is shown that the contact area is not centered with respect to the mean line of the rollers cross-section. In fact, depending on the translational speed $V$, the contact area may exhibit a certain degree of eccentricity $e$ (see [12]), due to the delay in the viscoelastic response of the material. In this regard, we observe that, within the contact strip $\Omega$, the layer displacement must copy the profile of the rigid cylinder $v(x)=R \sin \left[\cos ^{-1}(x / R)\right]$. Therefore, for $x \in \Omega$, Equation (1) can be rewritten as a Fredholm equation of the first kind

$$
\Delta-\Lambda[1-v(x+e)]=-\int_{-a}^{a} \Theta_{V}(x-s) p(s) d s
$$

where $\Lambda=R-\lambda^{-1} \int_{\lambda} v(x) d x$.

Interestingly, given $\Delta$ and $V$, Equation (9) can be inverted, allowing the calculation of the contact pressure distribution $p(x)$ as a function of the contact size $a$ and eccentricity $e$. Solving Equation (9) has been accomplished numerically, by exploiting the numerical procedure proposed in [44], which has been specifically developed for periodic contact mechanics, relying on a self-adaptive non-uniform discretization of the contact area. Indeed, to properly calculate the stress intensity factors, it is mandatory to solve the contact pressure field close to the contact leading and trailing edges at high 
resolution, thus a finer mesh is required. On the contrary, far from the contact edges, a coarser mesh allow to reduce the computational effort.

Dealing with adhesiveless contact conditions, the pressure distribution must remain positive (i.e., repulsive) everywhere, vanishing at the edge of the contact. Thus, for any given $\Delta$ and $V$, there is only one single choice of the couple $(a, e)$ fulfilling these conditions. Therefore, to calculate $a$ and $e$, following the authors of $[40,45-47]$, we also require that the "mode I" stress intensity factors vanish at the contact edges.

\section{Results and Discussion}

Results are obtained assuming $v=0.5$, and are shown in terms of dimensionless quantities. The following ones are therefore defined: $\tilde{h}=k h, \tilde{a}=a / R, \tilde{R}=R / \lambda, \tilde{p}=2\left(1-v^{2}\right) p /\left(E_{0} k R\right), \zeta=V \tau k$, and $\tilde{\Delta}=\Delta / \Lambda$. It is well known that in cases such as the one under investigation, in which the contact area is not negligibly small in comparison with the thickness of the deformable (e.g., viscoelastic) layer, the thickness $h$ strongly affects the results. For this reason, some results are shown for a couple of values of $\tilde{h}$ to discuss the influence of the layer thickness on the contact quantities, whereas the limiting case of contact with rigid indenters and a viscoelastic half-plane (i.e., $\tilde{h}=\infty$ ) can be found elsewhere in the literature [41].

Figure 2 shows the contact between a rigid indenter made with equally spaced cylinders of dimensionless radius $\tilde{R}$ and a viscoelastic layer with dimensionless thickness $\tilde{h}$, for two different values of the layer thickness, namely $\tilde{h}=1$ (Figure 2a) and $\tilde{h}=0.8$ (Figure 2b). The layer is pressed against the indenter by a uniform pressure. The schematic picture backs in mind the belt of a conveyor pressed against idle rollers by, for example, the distributed weight of some granular material. The layer has a leftward relative velocity with respect to the indenter and there is no friction at the interface between the cylinder and the belt. Because of the viscoelastic behavior of the layer, the area of contact with each cylinder is not symmetrically distributed with respect to the vertical symmetry line of each cylinder, but it is slightly displaced on the right side of the indenter. Figure 2 shows that the thinner layer is more compliant, thus the contact area is larger and, more evidently, the bending deflection of the layer is much more evident. This apparently trivial consideration is very important to understand the results discussed below.

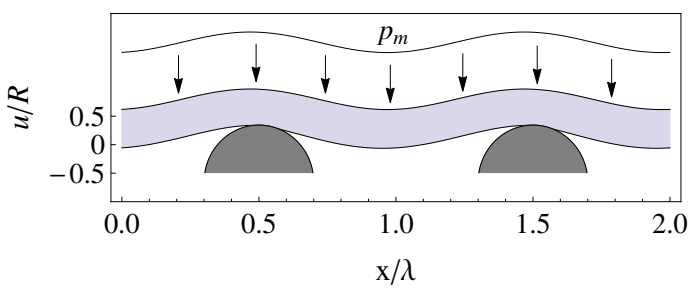

(a)

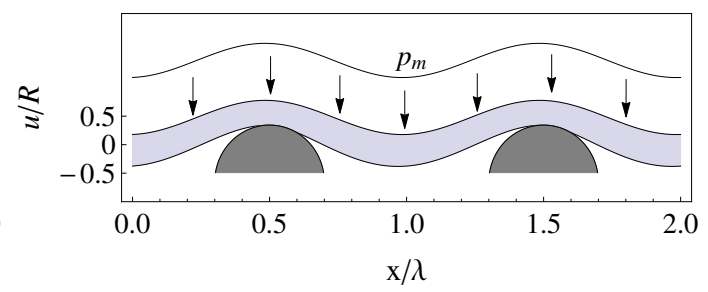

(b)

Figure 2. Schematic pictures of the viscoelastic layer with finite thickness and free boundary condition on the upper surface indented by two equal cylinders of radius $\mathrm{R}$ and spacing $\lambda$ on the lower surface, under the effect of an imposed constant pressure distribution on the upper surface of the layer: (a) for $\tilde{h}=1$; and $(\mathbf{b})$ for $\tilde{h}=0.8$.

It is worth noting that typical belt conveyors present a certain belt pre-tension, which is usually sustained by the stiffer metallic or fiber belt core. Due to the belt curvature between two successive rollers, such membrane pre-stresses give rise to a large deformation effect, increasing the bending stiffness of the belt, and in turn reducing the overall bending at fixed load. This effect is not considered in our model, which moves in the framework of small-deformations linear viscoelasticity. However, the results presented herein in terms of viscoelastic friction are not qualitatively affected by this assumption, because the pre-stress is not cyclically varying (i.e., it is constant), thus does not give rise to any viscoelastic dissipation. On the other hand, its effect in the real-world is to increase the 
apparent bending stiffness of the belt, thus a quantitive mismatch is expected: at given value of mean load $p_{m}$, our model overestimates both the bending deflection of the belt and the contact area with the rollers (which is strictly related to the bending deflection in the free layer case, such as the one under investigation).

Figure 3 shows, for different values of dimensionless velocity $\zeta$ and given $\tilde{R}=0.4$, the dimensionless contact semi-width $\tilde{a}$ as a function of the dimensionless load $\tilde{p}_{m}$. Figure $3 \mathrm{a}$ is for $\tilde{h}=1$ and Figure $3 \mathrm{~b}$ is for $\tilde{h}=0.8$. In Figure $3 \mathrm{a}, \mathrm{b}$, it emerges that the increase of the contact patch size with load is very steep when the value of $\tilde{a}$ is below 0.5-0.8 (within the investigated range of tangential velocity), whereas it slows down suddenly when such values are overcome, as $\tilde{a} \rightarrow 1$ is an unrealistic asymptotic contact limit, in agreement with the authors of [40]. The effect of increasing the tangential velocity is to reduce the contact area, due to the viscoelastic stiffening of the material, and also to reduce the threshold value of $\tilde{a}$ which determines the transition from compliant to stiff behavior. Finally, reducing the thickness of the layer (Figure 3b), the contact compliance noticeably increases throughout the whole load range. Such a result is well expected under load controlled conditions, since a reduction of the thickness of the viscoelastic layer increases the bending compliance, which enhances the attitude of the free layer to copy the indenter shape.

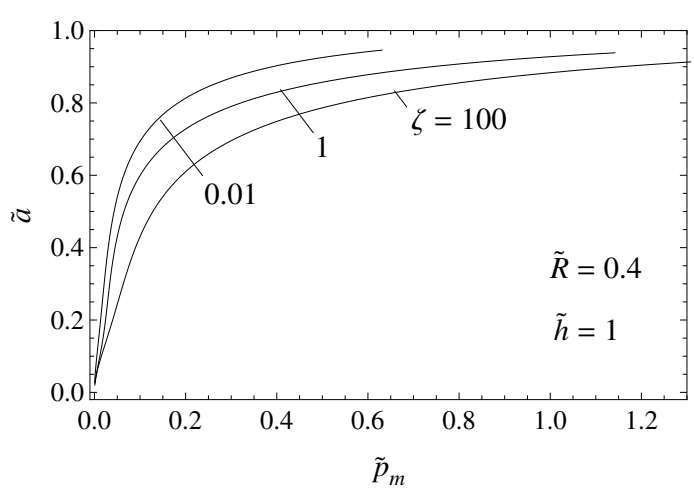

(a)

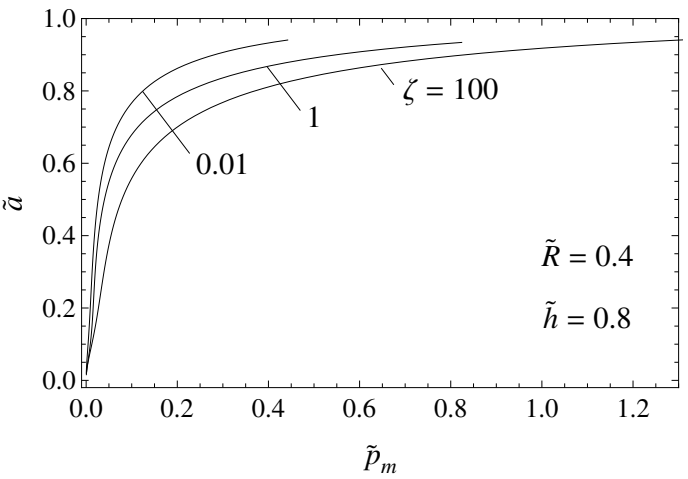

(b)

Figure 3. The dimensionless contact area $\tilde{a}$ as a function of the dimensionless load $\tilde{p}_{m}$ for one given value of the indenter radius $\tilde{R}=0.4$ and three values of the dimensionless tangential velocity $\zeta$ : (a) for $\tilde{h}=1$; and $(\mathbf{b})$ for $\tilde{h}=0.8$.

Regardless of the specific shape of the viscoelastic body, under rolling contact conditions, a kind of mechanical keying occurs as a consequence of the viscoelastic dissipation within the bulk material, which makes contact spots asymmetrical even on symmetrically shaped asperities, as observed in Figure 2. Thus, even in the ideal case of vanishing Coulomb friction, a tangential load opposing the relative motion occurs. This is the resultant of the projection of the local contact forces in the direction of the relative movement between the two bodies. Because of the dissipative origin of such force (viscoelastic dissipation in the bulk), it is usually referred to as viscoelastic friction force. Thus, a friction coefficient (rolling friction coefficient or indentation rolling resistance coefficient) can be defined as the ratio between the friction force and the normal load:

$$
\mu=\frac{F_{T}}{F_{N}}=\frac{\tau_{m}}{p_{m}}
$$

where $\tau_{m}=-\frac{1}{\lambda} \int_{\Omega} p(x) u^{\prime}(x) d x$ is the average "tangential" stress, $F_{T}=\tau_{m} b \lambda$ and $F_{N}=p_{m} b \lambda$, and $b$ is the tranverse width of the system.

The frictional behavior of the viscoelastic systems strongly depends on the excitation frequency $\omega$, through the viscoelastic complex modulus 


$$
E(\omega)=E_{0}+E_{1} \frac{i \omega \tau}{1+i \omega \tau}
$$

Indeed, at very small tangential velocity (i.e., at very small $\omega$ ), although the viscoelastic material is softer $\left(E(\omega) \approx E_{0}\right)$ and, in turn, the contact area is relatively large, the time-dependent layer deformation occurs sufficiently slow to allow the material to elastically deform and recover the original shape, thus leading to almost symmetrical distributions of contact pressure and to almost symmetrical contact area. Since the viscoelastic dissipation depends on the the cyclic deformation of the material, under this condition, we expect a small friction force and friction coefficient. On the other extreme, when the tangential velocity is very large (i.e., at very large $\omega$ ), the elastic modulus of the layer increases $\left(E(\omega) \approx E_{\infty}\right)$ and, under load controlled conditions, the contact area reduces compared to the low speed results. Since the amount of viscoelastic material involved in the cyclic deformation is roughly proportional to the contact area size, we have that at high velocity, small friction is expected as well. At intermediate values of tangential velocity, instead, the friction coefficient follows a characteristic bell-like curve with a pronounced local maximum. The maximum dissipation occurs when the excitation frequency $\omega$ maximizes the ratio $\operatorname{Im}[E(\omega)] /|E(\omega)|$. From Equation (10), we have that such a condition implies that $\omega \tau \approx 1$.

The friction coefficient $\mu$ is shown in Figure 4 as a function of the dimensionless tangential velocity for different values of the dimensionless radius $\tilde{R}$ of the rollers. In Figure $4 \mathrm{a}$, the dimensionless thickness of the layer is $\tilde{h}=1$ and in Figure $4 \mathrm{~b} \tilde{h}=0.8$. A close look at Figure $4 \mathrm{a}, \mathrm{b}$ suggests that maximum friction occurs for $\zeta \approx 1$, even though the cylinder dimensionless radii $\tilde{R}$ may significantly affect the overall result, depending on the layer thickness. A possible explanation of such a difference can be found by relying on dimensional arguments. Indeed, in the case of a relatively thick viscoelastic layer (Figure 4a), the main deformation involves the contact area zone, as the bending deformation is negligible (notably the bending stiffness is proportional to $h^{3}$ ). Thus, the main excitation frequency of the viscoelastic material is related to the size of the contact $a$, and can be estimated as $\omega_{a} \sim 2 \pi V / a \approx$ $2 \pi V / R$, assuming $a \approx R$. Considering that $V=\zeta \lambda /(2 \pi \tau)$, one obtains that for a relatively thick layer the estimated dimensionless velocity which maximizes the friction coefficient is $\zeta_{a} \sim \tilde{R}$, thus it reduces by reducing $\tilde{R}$ (it is worth noting that in our physical problem $\tilde{R} \leq 0.5$ ). On the other hand, for relatively thin layers (Figure $4 \mathrm{~b}$ ) most of the dissipation occurs in the bulk of the layer as a consequence of the cyclic bending deformation. In this case, the main excitation frequency can be estimated as $\omega_{b} \sim 2 \pi V / \lambda$, thus similar arguments as those discussed before lead to estimate that the tangential velocity which maximizes the friction coefficient as $\zeta_{b} \sim 1$, disregarding the value of $\tilde{R}$. Finally, in Figure $4 \mathrm{a}, \mathrm{b}$, we also observe that the friction coefficient increases with decreasing $\tilde{h}$. This result is even clearer in Figure 5, where average "tangential" stress $\tau_{m}$ is shown as a function of the dimensionless layer thickness $\tilde{h}$, under given normal load $\tilde{p}_{m}$, tangential velocity $\zeta$ and indenter radius $\tilde{R}$. Figure 5 also shows a comparison with the case of moving indentation of a half-plane, which, of course, is approached asymptotically when $\tilde{h}$ is very large. The increase of average friction stress with decreasing $\tilde{h}$ is the consequence of the combined effect of increasing both the bending deformation and the contact area. Such a result is opposite to the one described in [41] where the case of confined viscoelastic layer is investigated. Indeed, in that case, the smaller is the thickness, the stiffer is the contact, leading to a reduction of the amount of material deformed under load and thus reducing the energy dissipation (down to zero for a layer with infinitesimal thickness).

For practical applications, it could be interesting to quantify the amount of energy dissipated (per unit time and in steady-state condition) by the viscoelastic loss. If the energy dissipated per unit time is calculated as $W_{d}=\tau_{m} \lambda b V=F_{T} V$, the related dimensionless quantity is $\tilde{W}_{d}=2\left(1-v^{2}\right) \tau W_{d} /\left(E_{0} R \lambda b\right)$. Figure 6 shows $\tilde{W}_{d}$ as a function of the dimensionless relative velocity $\zeta$ for two values of the dimensionless radius of the indenter, namely $\tilde{R}=0.1$ and $\tilde{R}=0.3$. Figure 6a shows $\tilde{h}=1$ and Figure $6 \mathrm{~b}$ shows $\tilde{h}=0.8$. At small velocity values the dissipation vanishes since the layer deforms almost elastically. In the range $0.1<\zeta<10$, there is a sudden increase of the dissipated energy before 
it reaches plateaux for $\zeta>10$. Thus, in applications where the energy dissipation must be minimized, it is very important to design the device keeping $\zeta<0.1$. Remembering that $\zeta=V \tau k$ and considering that $V$ in general can not be set arbitrarily since it depends on the specific application, engineering design guidelines can be inferred aiming at tuning the relaxation time of the viscoelastic material $\tau$ and the spacing $\lambda$ of the cylinders to keep $\zeta$ within the desired range. Figure 6 also shows that the effect of reducing the cylinder radius is to increase dissipated energy per unit time and that the reduction of adimensionalized thickness has a similar effect. In both cases, such a behavior can be ascribed to the increase of layer bending deformation.

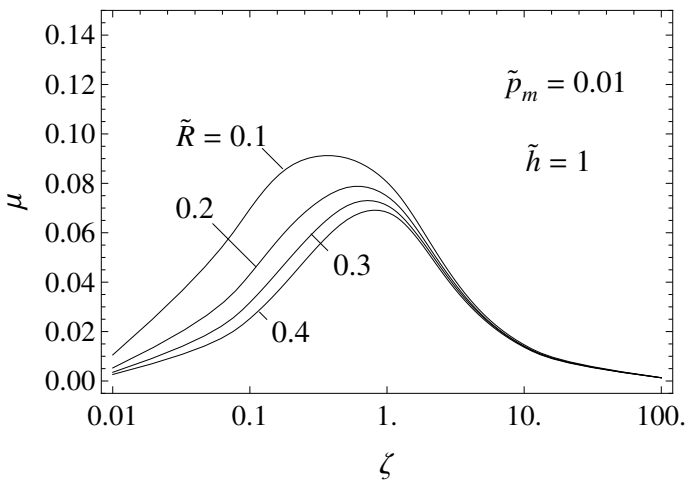

(a)

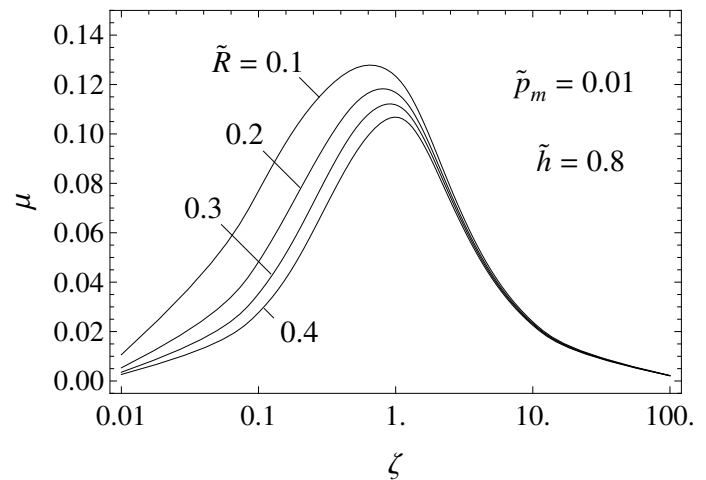

(b)

Figure 4. The friction coefficient as a function of the dimensionless tangential velocity $\zeta$ under load controlled conditions $\left(\tilde{p}_{m}=0.13\right)$ and four different values of indenter radii $\tilde{R}$. The finite thickness of the viscoelastic layer is: (a) $\tilde{h}=a$; and (b) $\tilde{h}=0.8$.

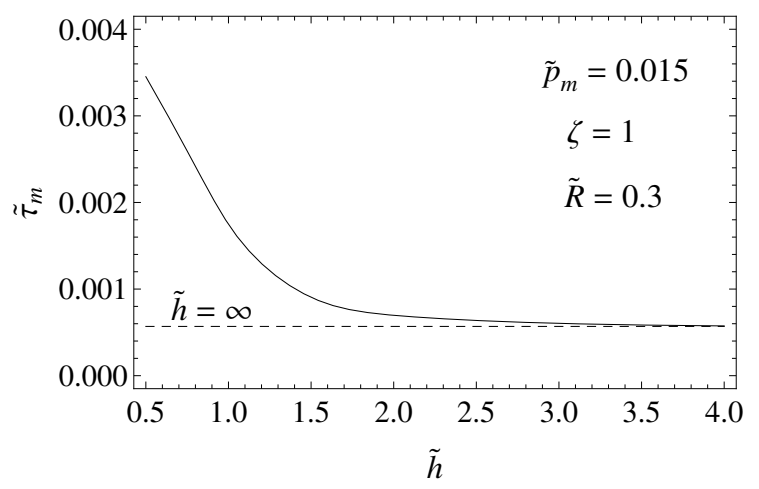

Figure 5. The average non-dimensional tangential load for given average contact pressure $\tilde{p}_{m}$, cylinder radius $\tilde{R}$ and non-dimensional velocity $\zeta$, as a function of the thickness of the viscoelastic layer $\tilde{h}$. The dashed line represents the results obtained in the half-space limit, corresponding to $\tilde{h}=\infty$.

The adimensionalized energy dissipated per unit time is also shown in Figure 7 as a function of the relative velocity $\zeta$ at several pressure $\tilde{p}_{m}$ values. We observe that increasing the load has two effects: (i) increasing the system deformations; and (ii) increasing the amount of material involved in the cyclic deformation. Indeed, Figure 7 shows that, increasing the load always leads to an increase of the energy dissipation, regardless of the tangential velocity. Further, in the plateaux region, it can be seen that the effect of increasing the load is more than linear since, when the load is doubled, the energy dissipated per unit time is almost multiplied by four. The asymptotic behavior of the energy dissipated per unit time at increasing velocity is rigorously studied in [41]. The energy dissipation does not fall to zero even though the material approaches an elastic behavior when the speed is increased, because the viscoelastic friction force only asymptotically goes to zero, but its product with the velocity has a finite asymptotic value. 


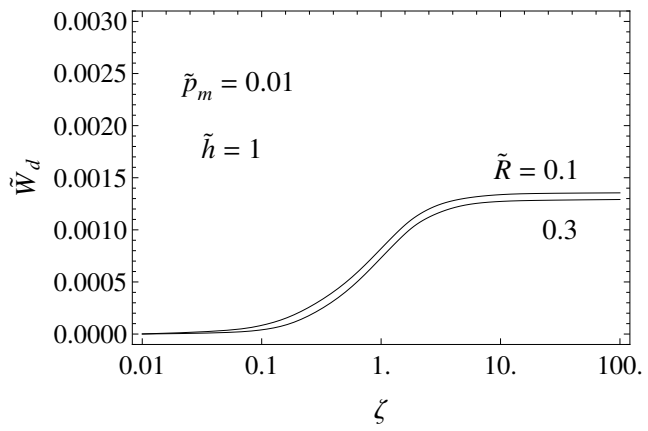

(a)

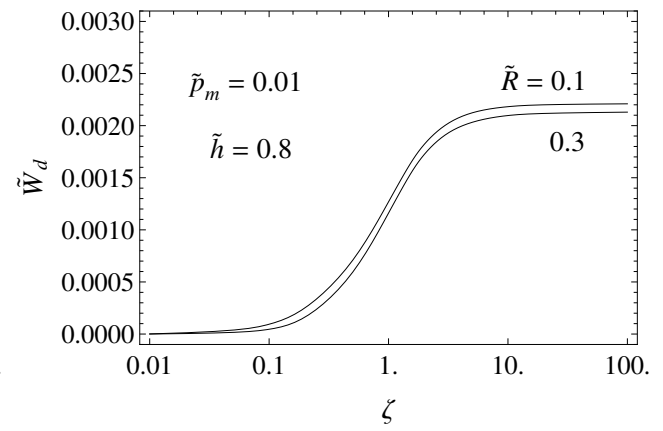

(b)

Figure 6. The dimensionless energy dissipated per unit time $\tilde{W}_{d}$ as a function of the dimensionless tangential velocity $\zeta$ under load controlled conditions (dimensionless pressure $\tilde{p}_{m}=0.001$ ): (a) for $\tilde{h}=1$; and (b) for $\tilde{h}=0.8$. Notice that the scale is the same in $(\mathbf{a}, \mathbf{b})$. Results are shown for two values of the radius of curvature of the indenter.

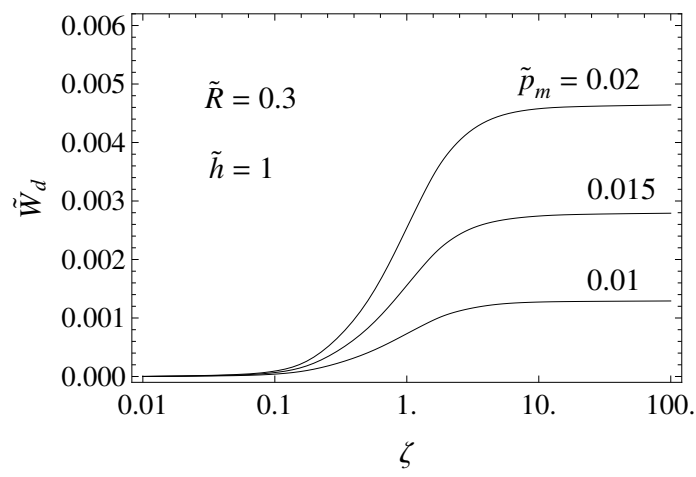

Figure 7. The non-dimensional energy dissipated (per unit time) $\tilde{W}_{d}$ as a function of the non-dimensional tangential velocity at one given value of layer thickness $\tilde{h}$ and cylinder radius $\tilde{R}$ and for three different values of the non-dimensional average contact pressure $\tilde{p}_{m}$.

\section{Conclusions}

In this paper, the steady-state rolling contact between a periodic array of rigid cylinders and a free, finite-thickness layer of single-relaxation time viscoelastic material is investigated. The influences of the layer thickness, the material properties, the cylinders radii and spacing are analyzed. Besides, the effects of working parameters such as the load and the tangential velocity are also studied. As typical of viscoelasticity, the system behavior is dominated by the velocity. At very low and very high velocities, the system behaves elastically, even though, in the very low limit, the material is more compliant, thus the deformations are larger, whereas, in the very high limit, the system is stiffer. At intermediate range of velocity values, viscous effects dominate and typical characteristics of the viscoelastic contacts are emphasized. In particular, the contact area is evidently non-symmetrical and the contact pressures have a resultant force with a quite large component opposing the relative motion between the cylinders and the layer: a viscoelastic friction arises. The curve of viscoelastic friction vs. velocity has a typical bell-like shape, with a local maximum in the intermediate velocity range. The location of the local maximum depends on the material relaxation time. This force, also known as indentation rolling resistance, is important in many applications, for example in belt conveyors. In such devices, it is one of the largest contributes to energy consumption. The energy dissipated per unit time is thus also studied as a function of the tangential velocity. It is small until the value of the adimensionalized velocity is below a threshold (about equal to 0.1 ), which is only mildly dependent on the applied load. It is also shown how the dissipation is nonlinearly affected by the applied load, and that increasing the load very rapidly increases the dissipated energy per unit time. In addition, the effect of the system geometrical and material parameters can be opportunely tuned to 
enhance the component functionality. For example, increasing the thickness of the layer or the cylinder radii at given load, reduces the viscoelastic friction and the related energy dissipation. On the other hand, at large thickness values, modifying the cylinder radii changes also the velocity profile of the viscoelastic friction force, since the local maximum is shifted upwards and towards smaller velocities as the cylinder radius is decreased. Instead, at small values of thickness, the shift happens only upwards, thus it is here shown how the influence of geometrical parameters is somehow interrelated in a complicated fashion with the material properties.

Author Contributions: All authors contributed equally to this work.

Funding: N.M. thanks the European Union, as this project received funding from the European Union's Horizon 2020 research and innovation programme under the Marie Skłodowska-Curie grant agreement No. 845756.

Conflicts of Interest: The authors declare no conflict of interest.

\section{References}

1. Ludema, K.; Tabor, D. The friction and visco-elastic properties of polymeric solids. Wear 1966, 9, 329-348. [CrossRef]

2. Zhou, X.; Yu, D.; Shao, X.; Zhang, S.; Wang, S. Research and applications of viscoelastic vibration damping materials: A review. Compos. Struct. 2016, 136, 460-480. [CrossRef]

3. Faisca, R.G.; Magluta, C.; Roitman, N. Experimental characterization of viscoelastic materials as vibration dampers. J. Eng. Mech. 2001, 127, 959-962. [CrossRef]

4. Quaroni, L.; Chumanov, G. Preparation of polymer-coated functionalized silver nanoparticles. J. Am. Chem. Soc. 1999, 121, 10642-10643. [CrossRef]

5. Hunter, S. The rolling contact of a rigid cylinder with a viscoelastic half space. J. Appl. Mech. 1961, 28, 611-617. [CrossRef]

6. Hager, M.; Hintz, A. The energy-saving design of belts for long conveyor systems. Bulk Solids Handl. 1993, 13, 749-749.

7. O'Shea, J.I.; Wheeler, C.A.; Munzenberger, P.J.; Ausling, D.G. The influence of viscoelastic property measurements on the predicted rolling resistance of belt conveyors. J. Appl. Polym. Sci. 2014, 131. [CrossRef]

8. Goryacheva, I.G. Contact Mechanics in Tribology; Springer Science \& Business Media: Berlin/Heidelberg, Germany, 2013; Volume 61.

9. Batra, S.; Ling, F. On deformation friction and interface shear stress in viscoelastic-elastic layered system under a moving load. Asle Trans. 1967, 10, 294-301. [CrossRef]

10. Grosch, K. The relation between the friction and visco-elastic properties of rubber. Proc. R. Soc. Lond. Ser. A Math. Phys. Sci. 1963, 274, 21-39.

11. Persson, B.N. Theory of rubber friction and contact mechanics. J. Chem. Phys. 2001, 115, 3840-3861. [CrossRef]

12. Menga, N.; Putignano, C.; Carbone, G.; Demelio, G. The sliding contact of a rigid wavy surface with a viscoelastic half-space. Proc. R. Soc. A Math. Phys. Eng. Sci. 2014, 470, 20140392. [CrossRef]

13. PANEK, C.; Kalker, J. Three-dimensional contact of a rigid roller traversing a viscoelastic half space. IMA J. Appl. Math. 1980, 26, 299-313. [CrossRef]

14. Kalker, J. Viscoelastic multilayered cylinders rolling with dry friction. J. Appl. Mech. 1991, 58, 666-679. [CrossRef]

15. Miftakhova, A. Contact Problems for Rolling with Slip for Viscoelastic Solids. J. Frict. Wear 2018, 39, 55-61. [CrossRef]

16. Le Tallec, P.; Rahler, C. Numerical models of steady rolling for non-linear viscoelastic structures in finite deformations. Int. J. Numer. Methods Eng. 1994, 37, 1159-1186. [CrossRef]

17. Bugnicourt, R.; Sainsot, P.; Lesaffre, N.; Lubrecht, A. Transient frictionless contact of a rough rigid surface on a viscoelastic half-space. Tribol. Int. 2017, 113, 279-285. [CrossRef]

18. Padovan, J.; Kazempour, A.; Tabaddor, F.; Brockman, B. Alternative formulations of rolling contact problems. Finite Elem. Anal. Des. 1992, 11, 275-284. [CrossRef]

19. Padovan, J. Finite element analysis of steady and transiently moving rolling nonlinear viscoelastic structure-I. Theory. Comput. Struct. 1987, 27, 249-257. [CrossRef] 
20. Carbone, G.; Putignano, C. A novel methodology to predict sliding and rolling friction of viscoelastic materials: Theory and experiments. J. Mech. Phys. Solids 2013, 61, 1822-1834. [CrossRef]

21. Nasdala, L.; Kaliske, M.; Becker, A.; Rothert, H. An efficient viscoelastic formulation for steady-state rolling structures. Comput. Mech. 1998, 22, 395-403. [CrossRef]

22. Schapery, R.A. On the characterization of nonlinear viscoelastic materials. Polym. Eng. Sci. 1969, 9, $295-310$. [CrossRef]

23. Odegard, G.; Gates, T.; Herring, H. Characterization of viscoelastic properties of polymeric materials through nanoindentation. Exp. Mech. 2005, 45, 130-136. [CrossRef]

24. Carbone, G.; Lorenz, B.; Persson, B.; Wohlers, A. Contact mechanics and rubber friction for randomly rough surfaces with anisotropic statistical properties. Eur. Phys. J. E 2009, 29, 275-284. [CrossRef] [PubMed]

25. Martina, D.; Creton, C.; Damman, P.; Jeusette, M.; Lindner, A. Adhesion of soft viscoelastic adhesives on periodic rough surfaces. Soft Matter 2012, 8, 5350-5357. [CrossRef]

26. Braat, G.; Kalker, J. Theoretical and experimental analysis of the rolling contact between two cylinders coated with multilayered, viscoelastic rubber. WIT Trans. Eng. Sci. 1970, 1, 8.

27. Padovan, J.; Paramadilok, O. Transient and steady state viscoelastic rolling contact. Comput. Struct. 1985, 20, 545-553. [CrossRef]

28. Persson, B. Rolling friction for hard cylinder and sphere on viscoelastic solid. Eur. Phys. J. E 2010, 33, 327-333. [CrossRef] [PubMed]

29. Goryacheva, I.; Sadeghi, F. Contact characteristics of a rolling/sliding cylinder and a viscoelastic layer bonded to an elastic substrate. Wear 1995, 184, 125-132. [CrossRef]

30. Johnson, K.L.; Johnson, K.L. Contact Mechanics; Cambridge University Press: Cambridge, UK, 1987.

31. Goryacheva, I.; Miftakhova, A. Modelling of the viscoelastic layer effect in rolling contact. Wear 2019, 430, 256-262. [CrossRef]

32. Torskaya, E.V.; Stepanov, F.I. Effect of surface layers in sliding contact of viscoelastic solids (3-D model of material). Front. Mech. Eng. 2019, 5, 26. [CrossRef]

33. Menga, N.; Afferrante, L.; Carbone, G. Adhesive and adhesiveless contact mechanics of elastic layers on slightly wavy rigid substrates. Int. J. Solids Struct. 2016, 88, 101-109. [CrossRef]

34. Menga, N. Rough frictional contact of elastic thin layers: The effect of geometrical coupling. Int. J. Solids Struct. 2019, 164, 212-220. [CrossRef]

35. Jonkers, C. The indentation rolling resistance of belt conveyors: A theoretical approach. Fördern Und Heb. 1980, 30, 312-317.

36. Lyubicheva, A. Analysis of the mutual influence of contact spots in sliding of the periodic system of asperities on a viscoelastic base of the winkler type. J. Frict. Wear 2008, 29, 92-98. [CrossRef]

37. Persson, B. Contact mechanics for layered materials with randomly rough surfaces. J. Phys. Condens. Matter 2012, 24, 095008. [CrossRef] [PubMed]

38. Scaraggi, M.; Persson, B. The effect of finite roughness size and bulk thickness on the prediction of rubber friction and contact mechanics. Proc. Inst. Mech. Eng. Part C J. Mech. Eng. Sci. 2016, 230, 1398-1409. [CrossRef]

39. Naghieh, G.; Jin, Z.; Rahnejat, H. Contact characteristics of viscoelastic bonded layers. Appl. Math. Model. 1998, 22, 569-581. [CrossRef]

40. Menga, N.; Afferrante, L.; Carbone, G. Effect of thickness and boundary conditions on the behavior of viscoelastic layers in sliding contact with wavy profiles. J. Mech. Phys. Solids 2016, 95, 517-529. [CrossRef]

41. Menga, N.; Foti, D.; Carbone, G. Viscoelastic frictional properties of rubber-layer roller bearings (RLRB) seismic isolators. Meccanica 2017, 52, 2807-2817. [CrossRef]

42. Menga, N.; Afferrante, L.; Demelio, G.; Carbone, G. Rough contact of sliding viscoelastic layers: Numerical calculations and theoretical predictions. Tribol. Int. 2018, 122, 67-75. [CrossRef]

43. Persson, B. On the elastic energy and stress correlation in the contact between elastic solids with randomly rough surfaces. J. Phys. Condens. Matter 2008, 20, 312001. [CrossRef]

44. Carbone, G.; Mangialardi, L. Analysis of the adhesive contact of confined layers by using a Green's function approach. J. Mech. Phys. Solids 2008, 56, 684-706. [CrossRef]

45. Charmet, J.C.; Barquins, M. Adhesive contact and rolling of a rigid cylinder under the pull of gravity on the underside of a smooth-surfaced sheet of rubber. Int. J. Adhes. Adhes. 1996, 16, 249-254. [CrossRef] 
46. Shull, K.R.; Ahn, D.; Mowery, C.L. Finite-size corrections to the JKR technique for measuring adhesion: soft spherical caps adhering to flat, rigid surfaces. Langmuir 1997, 13, 1799-1804. [CrossRef]

47. Carbone, G.; Mangialardi, L. Adhesion and friction of an elastic half-space in contact with a slightly wavy rigid surface. J. Mech. Phys. Solids 2004, 52, 1267-1287. [CrossRef]

(C) 2019 by the authors. Licensee MDPI, Basel, Switzerland. This article is an open access article distributed under the terms and conditions of the Creative Commons Attribution (CC BY) license (http://creativecommons.org/licenses/by/4.0/). 